\title{
Role of surface plasmons in laser-induced THz generation from metals
}

\author{
I. V. Oladyshkin, D. A. Fadeev, V. A. Mironov \\ Institute of Applied Physics of the Russian Academy of Sciences, Nizhny Novgorod, Russia \\ oladyshkin@gmail.com
}

\section{Introduction}

The paper is devoted to the analysis and preliminary theoretical interpretation of the last experimental results in laser-induced $\mathrm{THz}$ generation from metal structures. The main attention will be paid to metal gratings where resonant excitation of surface plasmons by the incident laser pulse is possible. Despite not a record efficiency level, study of optical-to-THz conversion on metal surfaces is relevant because of future applications in structural diagnostics and due to the fundamental interest in transformation mechanisms. Now there is no clear microscopic theory which is able to interpret the process of $\mathrm{THz}$ generation by metal gratings; in particular, the role of surface plasmons is not understood.

The first experimental measurements of $\mathrm{THz}$ signal from metal grating were performed more than 10 years ago [1]. In general, the strong influence of surface plasmon excitation on the $\mathrm{THz}$ generation was experimentally proved for different experimental conditions [1-3]. In the typical case a smooth resonant dependence of $\mathrm{THz}$ signal energy on the incidence angle is observed, where the resonant angle corresponds to the synchronism between the surface plasmon (on the optical frequency) and one of the diffraction waves $[1,2]$. In the same time, one of the most complicated experimental features is non-quadratic dependence of the $\mathrm{THz}$ radiation energy on the optical fluence.

\section{Mechanisms of plasmon-assisted THz generation}

There are several possible microscopic mechanisms of plasmon-enhanced $\mathrm{THz}$ generation which were proposed before. The first one is based on the emission and acceleration of electrons above the surface, the second one - on the ponderomotive action of the plasmon field inside the metal, the third one takes into account thermal effects in the electronic gas.

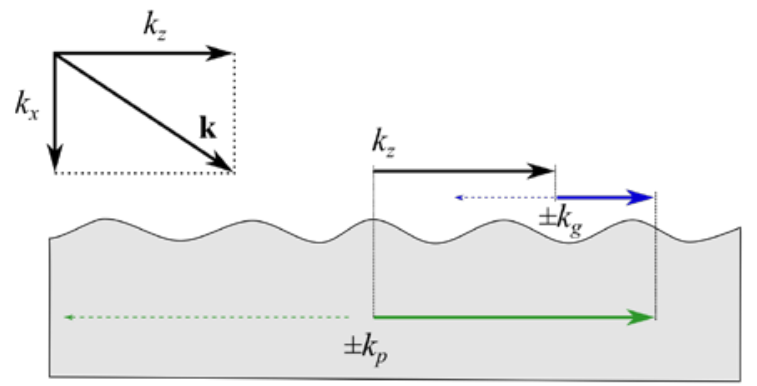

Fig. 1. Synchronism between the surface plasmon $k_{p}$ and incident optical wave $\mathbf{k}$ in the case of periodical grating with wavenumber $k_{g}: k_{z}+k_{g}=k_{p}$

The first mechanism was previously experimentally refuted in [3], where the authors irradiated struc- tured gold foil and observed $\mathrm{THz}$ emission from both incidence and rare side of the sample. Since electron emission and their acceleration into the substrate are impossible, the theory considering emitted electrons cannot be applicable in this case. The second mechanism can be also neglected since thermal nonlinear effects estimated to be stronger than ponderomotivelike ones in the case of metals [4]. So, here we will focus on the third (thermal) mechanism.

Thermal mechanism of $\mathrm{THz}$ generation for the case of flat metal surfaces was first proposed in [4], where analytical and numerical analysis was performed. Here we consider the same generation mechanism taking into account the possibility of surface plasmon excitation as an additional mechanism of the laser radiation absorption. Numerical model is based on Maxwell equations and hydrodynamical equations which describe electron gas motion and include heating by the laser field and heat diffusion.

\section{Resonance THz generation}

Well-known condition of synchronism between the surface plasmon wave and the optical wave above the metal grating is shown in the Fig. 1. In the first series of numerical calculations we study behavior of the $\mathrm{THz}$ response of the metal surface varying the grating period while the incidence angle was fixed $\left(30^{\circ}\right)$.

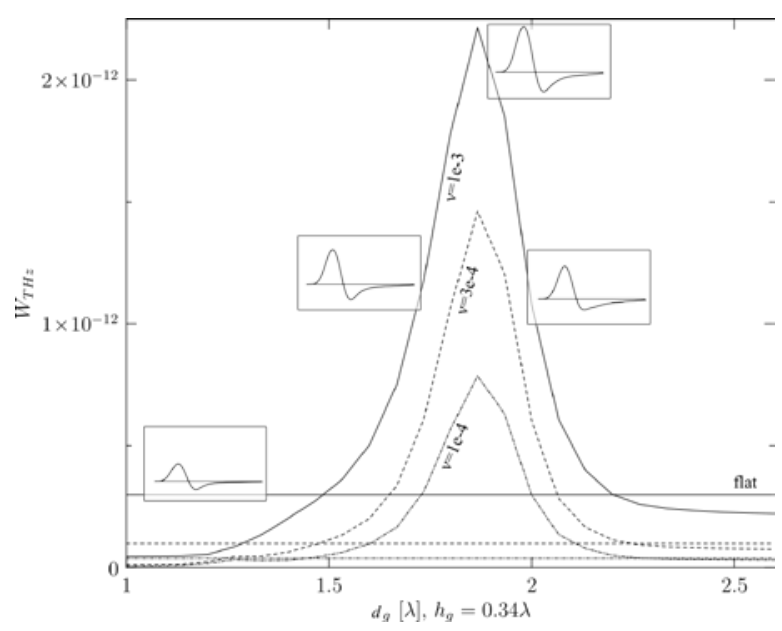

Fig. 2. Calculated dependence of the $\mathrm{THz}$ pulse energy on the grating period (angle of incidence $=30^{\circ}$, three different values of collision frequency are chosen). Insets: THz waveforms for different values of grating period.

Results presented in the Fig. 2 clearly indicate resonant character of the dependence (three curves correspond to three values of the scattering rate in metal, the grating period is measured in wavelengths 
of the incident optical pulse). Characteristic width of resonant curves equals to $10-15 \%$ of the resonant period which is in good agreement with experimental data from [2]. With the rise of collision frequency characteristic width also slightly increases.

\section{Optimal grating depth}

In the second series of numerical calculation we studied dependence of the conversion efficiency on the grating depth in the resonant point (see Fig. 3).

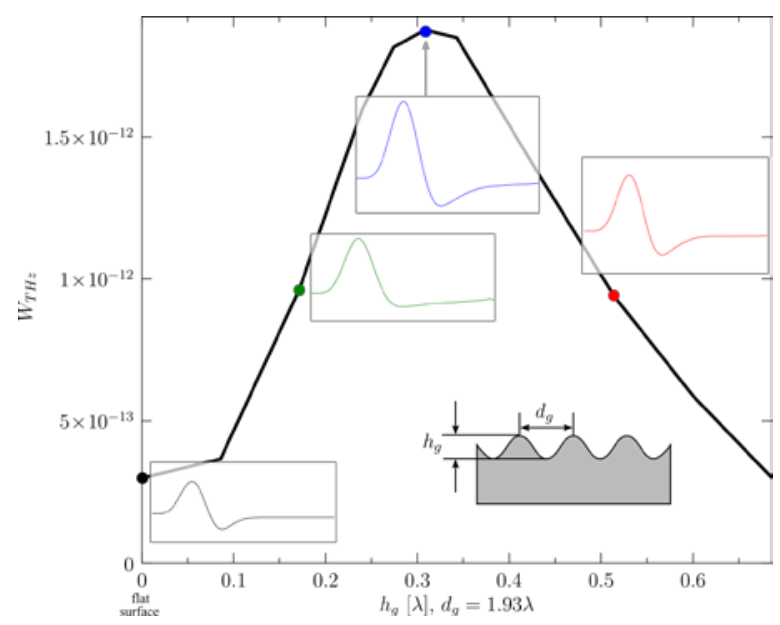

Fig. 3. Calculated dependence of the THz pulse energy on the grating depth (measured in the incident wavelengths). Angle of incidence equals to $30^{\circ}$. Insets: $\mathrm{THz}$ waveforms for different values of grating depth.

Obtained results are in good qualitative agreement with the experimental data from [3] where it was found that there is some optimal depth for THz generation (of about $150 \mathrm{~nm}$ for the optical wavelength 785 $\mathrm{nm}$ and incidence angle $\approx 45^{\circ}$ ). The further growth of the grating depth decreases $\mathrm{THz}$ signal. Quantitative comparison with the experiment requires modeling of the concrete grating profile (rectangular in most of cases). Calculated optimal value is about 0.3 wavelengths. The physical reason of this fact is a finite radiative lifetime of the surface plasmon which becomes shorter than the absorption time for too deep surface modulations.

To summarize, we perform numerical modeling of the $\mathrm{THz}$ signal generation process during the laser pulse reflection from the metal with periodically modified boundary. The key mechanism of low-frequency radiation generation is the medium polarization under the action of electronic temperature gradients. Here surface plasmon resonance can be treated an additional channel for the laser pulse absorption, so the resonance in absorption leads to rise of $\mathrm{THz}$ signal energy. Obtained numerical results (see Fig. 2 and Fig. 3) give a preliminary interpretation of the recent experimental data $[2,3]$

\section{Acknowledgements}

This work was supported by the Program of basic research of the Presidium of the Russian Academy of Sciences "Nonlinear dynamics: fundamental problems and applications". I.O. is grateful for support to the Foundation for the advancement of theoretical physics and mathematics "BASIS"

\section{References}

1. Welsh, G. H., Hunt, N. T., Wynne, K. Terahertz-pulse emission through laser excitation of surface plasmons in a metal grating // Phys. Rev. Lett. 2007. V. 98, No. 2. P. 026803.

2. Ramanandan, G. K. P., Ramakrishnan, G., Kumar, N., Adam, A. J. L., Planken, P. C. M., Emission of terahertz pulses from nanostructured metal surfaces // J. of Phys. D: Appl. Phys. 2014. V. 47, No. 37. P. 374003.

3. Garwe, F., Schmidt, A., Zieger, G., May, T., Wynne, K., Hübner, U., Zeisberger, M., Paa, W., Stafast, H., Meyer, H.-G., Bi-directional terahertz emission from gold-coated nanogratings by excitation via femtosecond laser pulses // Appl. Phys. B. 2011. V.102, No. 3. P. 551-554.

4. Oladyshkin, I. V., Fadeev, D. A., Mironov, V. A., Thermal mechanism of laser induced $\mathrm{THz}$ generation from a metal surface // J. of Optics 2015. V. 17, No. 7. P. 075502 